$\mathrm{XV}$.

\title{
CASES OF AURAL SUPPURATION PRESENTING IRREGULAR SYMPTOMS
}

\author{
By Eidward Bradford Dench, M. D.,
}

\section{NEW YORK.}

As it is the unusual in medicine, as in every other science, which interests, I beg to report very briefly three cases of aural suppuration which furnished rather unusual clinical pictures:

CASE 1. A boy about twelve years of age was admitted to my service at the New York Eye and Ear Infirmary, suffering from a chronic suppuration of the left middle ear. A radical operation was performed by one of my assistants and a primary graft applied. About four days after the operation the patient had a slight elevation in temperature and slight nystagmus toward the healthy side. The patient seemed to feel perfectly well and, aside from the temperature elevation and the slight nystagmus, presented no other unusual symptoms. The primary graft was removed and the wound packed. A slight infection of the superficial wound was present, and it was thought that this might explain the temperature. The wound was therefore freely opened and a wet dressing applied. For three succeeding days after the onset of the untoward symptoms, the temperature would be normal or nearly normal in the morning and would rise to $102^{\circ}$ or $103^{\circ}$ in the afternoon, the daily exacerbations becoming higher. Rotation showed the labyrinth of the affected side was dead. Blood cultures were negative. The original infection was streptococcic. Owing to the excursions in temperature, I was convinced that, in spite of negative blood cultures, we had to deal with a thombosis in the lateral sinus, and consequently asked my assistant to explore the sinus. This was done and the sinus was found to contain fluid blood, there being a very profuse hemorthage both from the lower and upper end of 
the sinus when this vessel was opened. The wound was packed in the ordinary manner and the patient returned to bed. The next day the temperature fell to normal in the morning, and the differential blood count, which had previously shown a high polymorphonuclear percentage, showed a diminished number of these cells. In the afternoon, however, the temperature again rose to $104^{\circ}$. The blood culture was still negative. Reasoning that a partial clot lying in the jugular bulb might give rise to these symptoms, although its size might be quite insufficient to cause any obstruction to the blood current, I removed the internal jugular on the day after the sinus was opened. The vein contained no clot, but seemed somewhat thickened at its upper portion. Unfortunately, the vein was not examined microscopically. The wound in the neck was closed by primary suture, a deep drain of folded rubber tissue being introduced throughout the entire length of the wound.

On the day after the jugular was removed the temperature rose to nearly $104^{\circ}$ in the afternoon. After that the temperature remained down steadily, and the patient made an uninterrupted recovery. A blood culture taken immediately after the removal of the jugular, showed the presence of streptococci. This is in direct opposition to the teaching so frequently laid down-that the removal of the jugular causes a disappearance of the streptococcemia.

This patient never had a streptococcemia until after the jugular was removed, and yet the removal of the jugular caused a complete abatement of all the symptoms of sinus thrombosis, and the patient went on to complete recovery. The wound in the neck healed throughout by first intention.

So much has been written recently regarding the value of the differential blood count and of blood cultures, in these cases, that it seems worth while to report a case of this character, in order to show that we must, after all, depend more upon our clinical symptoms than upon the laboratory findings. True, had we allowed this case to progress, we would undoubtedly have found streptococcemia, and would have removed the jugular at a later day. The best interests of the patient, however, were conserved by performing an early operation, and thus shutting off the focus of general infection before any general systemic involvement had occurred. 
Casf, 2. The patient was admitted to St. Luke's Hospital with a history of grip followed by a feeling of general malaise. Four days before his admission he suffered from severe pain in the right ear, and examination at the time of his admission to the hospital showed an acute inflammation of the right tympanic cavity. The right drum membrane was incised and considerable pus evacuated. The temperature, which was about $104^{\circ}$ on admission to the hospital, fell considerably after incision of the drum membrane, but rose again to $104^{\circ}$ the day after. The patient's temperature and general appearance suggested a beginning typhoid fever. The Widal reaction was indefinite on the first day. When I saw the patient, the right tympanic cavity was draining freely, there was very slight tenderness over the right mastoid, and the patient complained of moderate spontaneous pain in and about the ear. The polymorphonuclear count was somewhat elevated.

I advised observation for forty-eight hours, and if, at the end of this time, typhoid could not be diagnosed absolutely, that the mastoid should be opened and the sinus explored. I did not hear from the patient again until seventy-two hours after the time of my first visit, at which time the report was made that the Widal was less distinctive than before, and that the temperature had shown the marked fluctuations which had characterized the first two days' clinical history of the illness after admission to the hospital. I advised, therefore, the opening of the mastoid, exploration of the lateral sinus and excision of the internal jugular, provided no occlusive clot was found in the sinus. At the operation a few drops of pus were found in the mastoid cells. The sinus contained fluid blood and bled freely when opened. The jugular was excised in the usual manner. Three days afterwards the patient's temperature became normal, and he went on to a rapid recovery. In this case, the general history seemed to point to typhoid, and the patient's appearance certainly suggested a beginning, "continued" fever. The aural history was remarkably short for a case to develop symptoms of general sepsis, and for the first few days I was in doubt regarding the diagnosis. The absence of definite symptoms of typhoid, however, the presence of aural suppuration and the fluctuations in temperature determined the plan of procedure. The blood culture in this case was negative. 
In these two cases, the point of interest is the success of removing the internal jugular, in spite of the fact that the sinus seemed practically normal on inspection and palpation. Where a clot in the lateral sinus is of sufficient size to occlude the lumen of this vessel, I believe that opening the sinus and removing the clot may cause a cessation of all symptoms, and that this procedure, in cases of this character, may be carried out with hope of success in a certain proportion of cases without interference with the internal jugular vein. If, however, septic symptoms are present while the sinus seems normal, we can only suppose that these symptoms are caused by a small parietal clot, and occlusion of the sinus will not prevent general absorption. The only relief to be obtained, in these cases, is the removal of the internal jugular, thus shittting off the localized focus of infection from the general circulation.

It is probable that in both of the cases above narrated, the clot lay in the jugular bulb and was parietal.

CASE 3. A third case of interest was that of a patient upon whom I did a submucous operation about four weeks before the onset of his aural symptoms. The patient was in good general condition. The septal operation was exceedingly difficult, owing to the presence of adhesions between the displaced septum and the inferior turbinate of the occluded side. These adhesions made it necessary for me to tear the mucous membrane on the occluded side, in correcting the nasal obstruction. The patient did very well for about three weeks, when he contracted a severe cold in the head. One week after his cold he had an acute otitis upon the left side. This was mild in character, and the fluid evacuated, upon free incision of the drum membrane, which was performed eighteen hours after the inception of the acute otitis, was only a serous fluid, which showed no definite microorganisms, a few unclassified diplococci being present. The patient's temperature on admission was about $100^{\circ}$. It rose to $101^{\circ}$ after incision of the drum membrane, but fell to about $99^{\circ}$ on the following day. The discharge from the ear was very slight. On the day after the operation the temperature rose to $102^{\circ}$ in the evening, and on the second succeeding day, at the same hour in the evening, rose to $101^{\circ}$. The patient lived in a town where true intermittent fever is common and gave a history of wellmarked attacks of intermittent fever. For this reason, he 
was placed upon twenty grains of quinin daily. The diurnal excursions in temperature immediately became less, and the temperature did not go above $99^{\circ}$ or $991 / 2^{\circ}$ in the evening, for a period of three or four days. The patient, however, had profuse sweats, and did not seem to be gaining strength. Owing to the mild character of the febrile movement, no blood examination was made, as I did not feel that the plasmodium would be in evidence. Ten days aftter the patient's admission, the temperature suddenly rose to $102^{\circ}$, fell to normal, and the next day rose to $102^{\circ}$. Exxamination of the blood on this second day showed no plasmodium present; the polymorphonuclear percentage was 78 per cent, and the white blood count was $5,200,000$. The patient at this time had a slight bronchitis. I should have mentioned that the spleen was slightly enlarged at the first examination, and this sign was also present at the time of the last febrile attack. During this period, the middle ear suppuration had progressed favorably. The discharge had diminished in quantity, drainage was free, and there had never been more than the very slightest mastoid tenderness, and this was so ill-defined that it could scarcely be called "true mastoid tenderness." At the time of the acute attack there had been a hemorrhagic external otitis present, but this had gradually disappeared. In other words, the ear symptoms had progressed favorably for about ten days, and yet, at the end of this time, we had a rather pronounced febrile movement. The patient was examined by an internist, and several possibilities presented themselves. The temperature might be due to the slight bronchitis from which the patient was suffering, might be due to masked endocarditis, to sepsis from the ear, or a beginning typhoid. The relatively small leucocyte count seemed to be in favor of typhoid. Malarial infection was, naturally, ruled out, on account of the failure of the temperature to respond to large doses of quinin. On the second day of the febrile movement, the polymorphonuclear percentage fell to 68 per cent, and a blood culture taken twelve hours before was negative both for typhoid and streptococci. No medication, other than small dose of bromide, was employed. The patient's temperature fell to normal, and he made an uninterrupted recovery. What the exacerbations in temperature were due to, I do not know, but the case seems worthy of report, in conjunction with the other two cases mentioned above. 